\title{
On Brunn-Minkowski type inequality
}

\author{
Lewen Ji ${ }^{\mathrm{a}, \mathrm{b}}$, Zhenbing Zeng ${ }^{\mathrm{b}}$, Jingjing Zhong ${ }^{\mathrm{c}, *}$ \\ ${ }^{a}$ Department of Mathematics, East China University of Technology, Nanchang 330013, China. \\ ${ }^{b}$ Department of Mathematics, Shanghai University, Shanghai 200444, China. \\ ' School of Public Finance and Public Administration, Jiangxi University of Finance and Economics, Nanchang 330013, China.
}

Communicated by M. De La Sen

\begin{abstract}
The notion of Aleksandrov body in the classical Brunn-Minkowski theory is extended to that of Orlicz-Aleksandrov body in the Orlicz Brunn-Minkowski theory. The analogs of the Brunn-Minkowski type inequality and the first variations of volume are established via Orlicz-Aleksandrov body. We also make some considerations for the polar of Orlicz combination.
\end{abstract}

Keywords: Orlicz-Aleksandrov body, Brunn-Minkowski type inequality, Orlicz combination.

2010 MSC: 52A20, 52A40.

(C)2018 All rights reserved.

\section{Introduction and Preliminaries}

\subsection{Introduction}

The definition of Aleksandrov body in [1] was introduced by Aleksandrov to solve Minkowski problem in 1930. The Aleksandrov body given the relationship between the convex body containing the origin and the positive continuous functions and characterizes the convex body via the positive continuous functions. Aleksandrov body not only be used to solve Minkowski problem but also be applied to other areas of convex geometric analysis. However, the Brunn-Minkowski theory (see [2-5, 8, 10-17, 20]) plays an important role in convex geometric analysis.

The set of positive continuous functions on $S^{n-1}$ be denoted by $C^{+}\left(S^{n-1}\right)$ endowed with the topology derived from the max norm. Given a function $f \in C^{+}\left(S^{n-1}\right)$, the unique maximal element of

$$
\left\{K \in \mathcal{K}_{0}^{n}: h_{K}(u) \leqslant f(u), u \in S^{n-1}\right\},
$$

the Aleksandrov body associated with the positive continuous function $f \in \mathrm{C}^{+}\left(\mathrm{S}^{\mathrm{n}-1}\right)$ is denoted by

$$
K(f)=\max \left\{K \in \mathcal{K}_{0}^{n}: h_{K}(u) \leqslant f(u), u \in S^{n-1}\right\} .
$$

\footnotetext{
${ }^{*}$ Corresponding author

Email addresses: jilewen2008@163.com (Lewen Ji), zbzeng@shu.edu.cn (Zhenbing Zeng), 33515343@qq. com (Jingjing Zhong)

doi: $10.22436 /$ jnsa.011.06.03
}

Received: 2016-12-01 Revised: 2017-11-15 Accepted: 2018-03-01 
With the development of the Orlicz-Brunn-Minkowski theory. The Orlicz-Brunn-Minkowski theory originated with the work of Lutwak, Yang and Zhang in 2010. More precisely, Orlicz projection bodies and Orlicz centroid bodies were introduced by Lutwak, Yang, and Zhang in [15, 16], and they established the fundamental affine inequalities for these bodies. Haberl, Lutwak, Yang and Zhang in [7] dealt with the even Orlicz Minkowski problem. And most importantly, the general Aleksandrov body become a major goal. Here, we introduce a new geometric body: Orlicz-Aleksandrov body (as follows).

For $f \in C^{+}\left(S^{n-1}\right), \phi \in \mathcal{C}, K \in \mathcal{K}_{0}^{n}$ and $\varepsilon>-\min \left\{\phi\left(h_{K}\right) / \phi(f): u \in S^{n-1}\right\}$, define $h(\varepsilon, u)=$ $\phi^{-1}\left(\phi\left(h_{K}\right)+\varepsilon \phi(f)\right)$. We also define Orlicz-Aleksandrov body by

$$
\mathrm{K}\left(\mathrm{h}_{\mathrm{K}} \widehat{+}_{\phi} \varepsilon f\right)=\max \left\{\mathrm{K} \in \mathcal{K}_{0}^{\mathrm{n}}: \mathrm{h}_{\mathrm{K}}(\mathrm{u}) \leqslant \mathrm{h}(\varepsilon, \mathrm{u}), \mathrm{u} \in \mathrm{S}^{\mathrm{n}-1}\right\} .
$$

Throughout this paper, we set $\phi: \mathbb{R} \rightarrow[0, \infty)$ be a convex function such that $\phi(0)=0$ and $\phi$ be strictly increasing on $[0, \infty)$. The set is denoted by $\mathcal{C}$. It is easy to conclude from [18] that $\phi \in \mathcal{C}$ is continuous on $[0,+\infty)$ and the left derivative $\phi_{l}^{\prime}$ and right derivative $\phi_{\mathrm{r}}^{\prime}$ exist.

The purpose of this paper is to study the Aleksandrov body, we generalize the Brunn-Minkowski inequality for the Orlicz-Aleksandrov bodies associate with positive continuous functions and BrunnMinkowski type inequality for polar of Orlicz combination, as follows.

In Section 2, we compute the Orlicz first variations of volume and obtain their integral representation.

Theorem 1.1. Let $\mathrm{K} \in \mathcal{K}_{0}^{\mathrm{n}}$ and $\mathrm{f} \in \mathrm{C}^{+}\left(\mathrm{S}^{\mathrm{n}-1}\right)$, then, for $\phi \in \mathcal{C}$,

$$
\lim _{\varepsilon \rightarrow 0^{+}} \frac{\left|K\left(h_{K} \widehat{+}_{\phi} \varepsilon f\right)\right|-|K|}{\varepsilon}=\int_{S^{n-1}} \phi(f) / \phi_{r}^{\prime}(h(K, u)) d S(K, u) .
$$

And the Brunn-Minkowski type inequality is generalized to the Orlicz setting.

Theorem 1.2. Let $\mathrm{f}, \mathrm{g} \in \mathrm{C}^{+}\left(\mathrm{S}^{\mathrm{n}-1}\right)$ and $\phi \in \mathcal{C}$, then for all $0<\lambda<1$,

$$
\left|\lambda f \widehat{+}_{\phi}(1-\lambda) g\right| \geqslant|f|^{\lambda}|g|^{(1-\lambda)} \text {. }
$$

In Section 3, we are mainly interesting in studying generalizations of the previous relation [5]. we extend the Brunn-Minkowski type inequality to the Orlicz combination of convex bodies, we prove the following result.

Theorem 1.3. Let $\mathrm{K}, \mathrm{L} \in \mathcal{K}_{0}^{\mathrm{n}}, \lambda \in(0,1)$ and $\phi \in \mathcal{C}$. Then

$$
\left|\left(\lambda \mathrm{K}+_{\phi}(1-\lambda) \mathrm{L}\right)^{*}\right| \leqslant\left|\phi^{-1}(1) \mathrm{K}^{*}\right|^{\lambda}\left|\phi^{-1}(1) \mathrm{L}^{*}\right|^{1-\lambda} .
$$

Suppose $\phi(t)=t^{p}, p \geqslant 1$. The above volume case was already obtained by $[8,11]$.

\subsection{Preliminaries}

We collect some basic facts about convex bodies that are needed in our paper.

Let $\mathrm{K}$ be a convex body (compact convex subset with nonempty interiors) in $\mathbb{R}^{\mathrm{n}}$. $\mathcal{K}^{\mathrm{n}}$ denotes the set of convex bodies in $\mathbb{R}^{n}$ and denote by $\mathcal{K}_{0}^{n}$ the set of convex bodies containing the origin as interior. $|K|, V_{1}(K, L)$ denoted volume and mixed volume, respectively. Support function $h_{K}$ of convex body is defined by

$$
h_{K}(u):=h(K, u)=\max _{u \in S^{n-1}}\{x \cdot u: x \in K\},
$$

where $x \cdot u$ denoted the inner product of $u$ and $x$ (see [11]).

The Minkowski addition with respect to $K$ and $L$ in $\mathbb{R}^{n}$ is defined by (see[10])

$$
a K+b L=\{a x+b y: x \in K, y \in L\}, \text { for all } a, b>0 .
$$

If $K, L \in \mathcal{K}_{0}^{n}$ can be defined as a convex body such that

$$
h_{a K+b L}(u)=a h_{K}(u)+b h_{L}(u) \text {, for all } u \in S^{n-1} \text {. }
$$


And Minkowski's mixed volume inequality

$$
\mathrm{V}_{1}(\mathrm{~K}, \mathrm{~L})^{\mathrm{n}} \geqslant|\mathrm{K}|^{\mathrm{n}-1}|\mathrm{~L}|,
$$

with equality holds if and only if $\mathrm{K}$ and $\mathrm{L}$ are homothetic.

For convex body $\mathrm{K} \in \mathcal{K}_{0}^{n}$, let $\mathrm{K}^{*}$ denotes the polar of the body $\mathrm{K}$. Namely,

$$
\mathrm{K}^{*}=\left\{x \in \mathrm{R}^{\mathrm{n}}: \mathrm{x} \cdot \mathrm{y} \leqslant 1 \text {, for all } \mathrm{y} \in \mathrm{K}\right\} \text {. }
$$

Obviously, we have $\mathrm{K}^{* *}=\mathrm{K}$. If $\mathrm{K} \in \mathcal{K}_{0}^{n}$, then the support and radial functions of $\mathrm{K}^{*}$ is defined by $\mathrm{h}\left(\mathrm{K}^{*}, \cdot\right)=\frac{1}{\rho(\mathrm{K}, \cdot)}$.

We now turn to the Orlicz addition, which is an extension of $L_{p}$-addition. Let $K, L \in \mathcal{K}_{0}^{n}, a, b>0$ and $\phi \in \mathcal{C}$. The Orlicz combination $\mathrm{aK}+_{\phi} \mathrm{bL}$ is the convex body with support function $[6,19]$

$$
h\left(a K+_{\phi} b L, x\right)=\inf \left\{\lambda>0: a \phi\left(\frac{h(K, x)}{\lambda}\right)+b \phi\left(\frac{h(L, x)}{\lambda}\right) \leqslant 1\right\} .
$$

Since $\phi$ is strictly increasing, then

$$
\lambda \rightarrow a \phi\left(\frac{h(K, x)}{\lambda}\right)+b \phi\left(\frac{h(L, x)}{\lambda}\right),
$$

is strictly decreasing. Therefore, $h\left(a K++_{\phi} b L, x\right)=\lambda_{0}$ if and only if

$$
a \phi\left(\frac{h(K, x)}{\lambda_{0}}\right)+b \phi\left(\frac{h(L, x)}{\lambda_{0}}\right)=1
$$

When $\phi(t)=t^{p}$, for all $p \geqslant 1$, the Orlicz combinationis precisely the $\mathrm{L}_{p}$ Minkowski combination $a K+p$ bL.

We say that $K$ be a star body about the origin, if $K$ has continuous and positive radial function $\rho(K, \cdot)$. The radial function of $K$, is defined by

$$
\rho(K, x)=\max \{\lambda \geqslant 0: \lambda x \in K\}, x \in \mathbb{R}^{n} \backslash\{0\} .
$$

The class of star bodies about the origin o in $\mathbb{R}^{n}$ is denoted by $\mathcal{S}_{0}^{n}$. Star body $\mathrm{K}$ can be uniquely determined by its radial function $\rho(K, \cdot)$. If $\lambda>0$, we get that

$$
\rho(K, \lambda x)=\frac{1}{\lambda} \rho(K, x) ; \rho(\lambda K, x)=\lambda \rho(K, x) .
$$

In order to maintain the consistency of the symbols in this paper, we redefine the dual Orlicz radial combination $a K \widetilde{+}_{\phi} b L(a, b>0)$. Let $K, L \in \mathcal{S}_{0}^{n}$ and $\phi \in \mathcal{C}$ by [21]

$$
\rho\left(a K \tilde{+}_{\phi} b L, x\right)=\sup \left\{\lambda>0: a \phi\left(\frac{\lambda}{\rho(K, x)}\right)+b \phi\left(\frac{\lambda}{\rho(L, x)}\right) \leqslant 1\right\}
$$

for all $x \in R^{n}$. Since $\phi$ is strictly increasing, then

$$
\lambda \rightarrow a \phi\left(\frac{\lambda}{\rho(K, x)}\right)+b \phi\left(\frac{\lambda}{\rho(L, x)}\right)
$$

is strictly increasing. Therefore, $\rho\left(a K \widetilde{+}_{\phi} b L, x\right)=\lambda_{0}$ if and only if

$$
a \phi\left(\frac{\lambda_{0}}{\rho(K, x)}\right)+b \phi\left(\frac{\lambda_{0}}{\rho(L, x)}\right)=1
$$


If $\phi(t)=t^{p}, p \geqslant 1$, then the Orlicz radial combination reduces to Lutwak's radial harmonic $L_{p^{-}}$ combination $a \mathrm{~K} \tilde{+}_{-p} \mathrm{bL}(\mathrm{a}, \mathrm{b}>0)$, that is,

$$
\rho\left(a K \widetilde{+}_{p} b L, x\right)^{-p}=a \rho(K, x)^{-p}+b \rho(L, x)^{-p} .
$$

According to Lemmas 3.5 and 4.1 in [21], it is easy to check that $K \widetilde{+}_{\phi} \varepsilon L \rightarrow K$, as $\varepsilon \rightarrow 0^{+}$. And

$$
\lim _{\varepsilon \rightarrow 0^{+}} \frac{\rho\left(K \widetilde{+}_{\phi} \varepsilon L, u\right)^{n}-\rho(K, u)^{n}}{\varepsilon}=-\frac{n}{\phi_{l}^{\prime}(1)} \phi\left(\frac{\rho(K, u)}{\rho(L, u)}\right) \rho(K, u)^{n}
$$

is uniform on $S^{n-1}$, where $\phi_{l}^{\prime}(1)$ denotes the left-continuous derivative of $\phi$ at 1 .

According to Theorem 4.1 in [21], we easily obtain the following results.

Let $K, L \in \mathcal{S}_{0}^{n}$ and $\phi \in \mathcal{C}$, then, for all $1 \leqslant i \leqslant n$,

$$
-\frac{\phi_{l}^{\prime}(1)}{n} \lim _{\varepsilon \rightarrow 0^{+}} \frac{\left|K \tilde{+}_{\phi} \varepsilon L\right|-|K|}{\varepsilon}=\frac{1}{n} \int_{S^{n-1}} \phi\left(\frac{\rho(K, u)}{\rho(L, u)}\right) \rho(K, u)^{n} d u .
$$

From the above equality, we can define the dual Orlicz mixed volume $\widetilde{V}_{\phi}(K, L)$ of $K, L \in \mathcal{S}_{0}^{n}$ by

$$
\widetilde{V}_{\phi}(K, L)=\frac{1}{n} \int_{S^{n-1}} \phi\left(\frac{\rho(K, u)}{\rho(L, u)}\right) \rho(K, u)^{n} d u
$$

If $\phi(t)=t^{p}, p \geqslant 1, \widetilde{V}_{\phi}(K, L)$ turns to $\widetilde{V}_{-p}(K, L)$ of the $L_{p}$-dual mixed volume of $K$ and $L$.

We also establish the following dual Orlicz-Minkowski inequality via a similar method in [21]. Suppose that $K, L \in \mathcal{S}_{0}^{n}$ and $\phi \in \mathcal{C}$, then

$$
\widetilde{\mathrm{V}}_{\phi}(\mathrm{K}, \mathrm{L}) \geqslant|\mathrm{K}| \phi\left(\frac{|\mathrm{K}|^{1 / \mathrm{n}}}{|\mathrm{L}|^{1 / \mathrm{n}}}\right) .
$$

If $\phi$ is strictly convex, equality holds if and only if $\mathrm{K}$ and $\mathrm{L}$ are dilates.

We further establish the following dual Orlicz-Brunn-Minkowski inequality: Let $K, L \in \mathcal{S}_{0}^{n}$ and $\phi \in \mathcal{C}$, then

$$
1 \geqslant a \phi\left(\left(\frac{\left|a K \widetilde{+}_{\phi} b L\right|}{|K|}\right)^{\frac{1}{n}}\right)+b \phi\left(\left(\frac{\left|a K \tilde{+}_{\phi} b L\right|}{|L|}\right)^{\frac{1}{n}}\right) .
$$

If $\phi$ is strictly convex, equality holds if and only if $\mathrm{K}$ and $\mathrm{L}$ are dilates.

\section{Aleksandrov body}

A function $h \in \mathrm{C}^{+}\left(\mathrm{S}^{\mathrm{n}-1}\right)$ defines a family $\left\{\mathrm{H}_{\mathrm{u}}\right\}_{\mathrm{S}^{n-1}}$ of hyperplanes

$$
\mathrm{H}_{\mathfrak{u}}=\left\{x \in \mathrm{R}^{\mathrm{n}-1}: x \cdot \mathrm{u}=\mathrm{h}(\mathrm{u})\right\}
$$

We should be interested in the intersection of the halfspaces that are associated $h$ via a family $\left\{H_{\mathfrak{u}}\right\}_{S^{n-1}}$. This gives the convex body

$$
K=\cap_{S^{n-1}}\left\{x \in R^{n-1}: x \cdot u \leqslant h(u)\right\} .
$$

Obviously,

$$
h_{K} \leqslant h .
$$

Aleksandrov also proved that the inverse spherical image of $K$ of the set

$$
\omega_{h}=\left\{u \in S^{n-1}: h(K, u)<h(u)\right\},
$$

be a singular boundary point of $K$. Since the set of singular boundary points of a convex body has (n1)-dimensional Hausdorff measure zero[18]. It follows that $S\left(K, \omega_{h}\right)=0$. Consequently, if $h \in C^{+} S^{n-1}$, 
then $h\left(K_{h}, u\right) \leqslant h(u)$ and $h\left(K_{h}, u\right)=h(u)$ almost everywhere with respect to the surface area $S\left(K_{h}, \cdot\right)$. Moreover, there are

$$
\left|K_{h}\right|=\frac{1}{n} \int_{S^{n-1}} h(u) d S\left(K_{h}, u\right) .
$$

The volume $|\mathrm{h}|$ of a function $\mathrm{h} \in \mathrm{C}^{+}\left(\mathrm{S}^{\mathrm{n}-1}\right)$ is defined as the volume of the Aleksandrov body associated with $h$. Since the Aleksandrov body associated with the support function $h_{K}$ of a convex body $K$ is the body $\mathrm{K}$ itself, we have

$$
\left|h_{\mathrm{K}}\right|=|\mathrm{K}| \text {. }
$$

In order to prove Theorem 1.1, we need the following convergence lemma of Aleksandrov: If the functions $f_{0}, f_{1}, \cdots \in C^{+}\left(S^{n-1}\right)$ have associated with Aleksandrov body $K_{0}, K_{1}, \cdots \in \mathcal{K}_{0}^{n}$ and $\lim _{n \rightarrow \infty} f_{n}=$ $f_{0}$, uniformly on $S^{n-1}$, then $\lim _{n \rightarrow \infty} K_{n}=K_{0}$. This gives $|\cdot|: C^{+}\left(S^{n-1} \rightarrow(0, \infty)\right.$ is continuous.

Proof of Theorem 1.1. We let $\mathrm{K}_{\varepsilon}$ denote the Aleksandrov body of $h(\varepsilon, \mathrm{u})$. Since

$$
\lim _{\varepsilon \rightarrow 0^{+}} h(\varepsilon, \cdot)=h(K, \cdot)
$$

uniformly on $S^{n-1}$, it follows that Aleksandrov's convergence lemma that $\lim _{\varepsilon \rightarrow 0^{+}} K_{\varepsilon}=K$. Hence, we conclude that

$$
\lim _{\varepsilon \rightarrow 0^{+}} S\left(K_{\varepsilon}, \cdot\right)=S(K, \cdot) \text {, weakly on } S^{n-1}
$$

and

$$
\lim _{\varepsilon \rightarrow 0^{+}} \frac{h(\varepsilon, u)-h(K, u)}{\varepsilon}=\frac{\phi(f(u))}{\phi_{r}^{\prime}(h(K, u))} \text {, uniformly on } S^{n-1} .
$$

According to Lemma 1 in [7], it easy to check that

$$
\lim _{\varepsilon \rightarrow 0^{+}} \frac{\left|K_{\varepsilon}\right|-|K|}{\varepsilon}=\int_{S^{n-1}} \phi(f) / \phi_{r}^{\prime}(h(K, u)) d S(K, u) .
$$

As desired.

In view of Theorem 1.1, it is hard to get the Minkowski type inequality via the first variation of volume. However, we define

$$
\widehat{\mathrm{V}}_{\phi}(\mathrm{K}, \mathrm{f})=\lim _{\varepsilon \rightarrow 0^{+}} \frac{\left|\mathrm{K}_{\varepsilon}\right|-|\mathrm{K}|}{\varepsilon}
$$

and obtain a lower bound as the following.

Theorem 2.1. Let $\mathrm{K} \in \mathcal{K}_{0}^{\mathrm{n}}$ and $\mathrm{f} \in \mathrm{C}^{+}\left(\mathrm{S}^{\mathrm{n}-1}\right)$, then, for $\phi \in \mathcal{C}$,

$$
\widehat{V}_{\phi}(K, f) \geqslant n|f|^{\frac{1}{n}}|K|^{\frac{n-1}{n}} \text {. }
$$

Proof. Since $\phi \in \mathcal{C}$, we know that $\phi^{-1}$ is strictly increase and concave function, suppose $0<\varepsilon<1$, it follows that, $K_{\varepsilon}$ as define in Theorem 2.1,

$$
\begin{aligned}
\left|K_{\varepsilon}\right| & =\frac{1}{n} \int_{S^{n-1}} h(\varepsilon, u) d S\left(K_{\varepsilon}, u\right) \\
& =\frac{1}{n} \int_{S^{n-1}} \phi^{-1}\left(\phi\left(h_{K}\right)+\varepsilon \phi(f)\right) d S\left(K_{\varepsilon}, u\right) \\
& \geqslant \frac{1}{n} \int_{S^{n-1}} \phi^{-1}\left((1-\varepsilon) \phi\left(h_{K}\right)+\varepsilon \phi(f)\right) d S\left(K_{\varepsilon}, u\right) \\
& \geqslant \frac{1}{n} \int_{S^{n-1}}\left[(1-\varepsilon) h_{K}+\varepsilon f\right] d S\left(K_{\varepsilon}, u\right) \\
& =(1-\varepsilon) V_{1}\left(K_{\varepsilon}, K\right)+\varepsilon V_{1}\left(K_{\varepsilon}, K_{f}\right) \\
& \geqslant(1-\varepsilon)\left|K_{\varepsilon}\right|^{\frac{n-1}{n}}|K|^{\frac{1}{n}}+\varepsilon\left|K_{\varepsilon}\right|^{\frac{n-1}{n}}|f|^{\frac{1}{n}}
\end{aligned}
$$


the above inequality implies

$$
\left|\mathrm{K}_{\varepsilon}\right|^{\frac{1}{n}} \geqslant(1-\varepsilon)|\mathrm{K}|^{\frac{1}{n}}+\varepsilon|f|^{\frac{1}{n}} .
$$

Thus,

$$
\begin{aligned}
\widehat{V}_{\phi}(K, f) & =\lim _{\varepsilon \rightarrow 0^{+}} \frac{\left|K_{\varepsilon}\right|-|K|}{\varepsilon} \\
& \geqslant \lim _{\varepsilon \rightarrow 0^{+}} \frac{\left[(1-\varepsilon)|K|^{\frac{1}{n}}+\varepsilon|f|^{\frac{1}{n}}\right]^{n}-|K|}{\varepsilon} \\
& =n|f| \frac{1}{n}|K|^{\frac{n-1}{n}} .
\end{aligned}
$$

This proves the theorem.

We next show that the Brunn-Minkowski type inequality for Orlicz-Aleksandrov body.

Theorem 2.2. Let $\mathrm{f}, \mathrm{g} \in \mathrm{C}^{+}\left(\mathrm{S}^{\mathrm{n}-1}\right)$ and $\phi \in \mathcal{C}$, then for all $0<\lambda<1$,

$$
\left|\lambda f \widehat{+}_{\phi}(1-\lambda) g\right|^{\frac{1}{n}} \geqslant \lambda|f|^{\frac{1}{n}}+(1-\lambda)|g|^{\frac{1}{n}} .
$$

Proof. Since $\phi \in \mathcal{C}$, we conclude that $\phi^{-1}$ is concave function, hence,

$$
\begin{aligned}
\left|\mathrm{K}\left(\lambda f \widehat{+}_{\phi}(1-\lambda) g\right)\right| & =\frac{1}{n} \int_{S^{n-1}} h\left(K\left(\lambda f \widehat{+}_{\phi}(1-\lambda) g\right), u\right) d S\left(K\left(\lambda f \widehat{+}_{\phi}(1-\lambda) g\right), u\right) \\
& =\frac{1}{n} \int_{S^{n-1}} \phi^{-1}(\lambda \phi(f)+(1-\lambda) \phi(g)) d S\left(K\left(\lambda f \widehat{+}_{\phi}(1-\lambda) g\right), u\right) \\
& \geqslant \frac{1}{n} \int_{S^{n-1}} \lambda f+(1-\lambda) g d S\left(K\left(\lambda f \widehat{+}_{\phi}(1-\lambda) g\right), u\right) \\
& \geqslant V_{1}\left(K\left(\lambda f \widehat{+}_{\phi}(1-\lambda) g\right), K_{f}\right)+V_{1}\left(K\left(\lambda f \widehat{+}_{\phi}(1-\lambda) g\right), K_{g}\right),
\end{aligned}
$$

the above inequality yields

$$
\left|\lambda f \widehat{+}_{\phi}(1-\lambda) g\right|^{\frac{1}{n}}=\left|K\left(\lambda f \widehat{+}_{\phi}(1-\lambda) g\right)\right|^{\frac{1}{n}} \geqslant \lambda|f|^{\frac{1}{n}}+(1-\lambda)|g|^{\frac{1}{n}} .
$$

This completes the proof.

Corollary 2.3. Let $\mathrm{K}, \mathrm{L} \in \mathcal{K}_{0}^{\mathrm{n}}$ and $\phi \in \mathcal{C}$, then for all $0<\lambda<1$,

$$
\left|\lambda K \widehat{+}_{\phi}(1-\lambda) L\right|^{\frac{1}{n}} \geqslant \lambda|K|^{\frac{1}{n}}+(1-\lambda)|L|^{\frac{1}{n}} .
$$

We also establish the proof of Theorem 1.2 as follows.

Proof of Theorem 1.2. According to Theorem 2.2 and arithmetic-geometric mean inequality, we obtain the desired result.

\section{Polar Set}

In this section, we get the relationship between Orlicz combination and Orlicz radial combination, and obtain Brunn-Minkowski type inequality for polar of Orlicz linear combination.

Lemma 3.1. Let $\mathrm{K}, \mathrm{L} \in \mathcal{K}_{0}^{\mathrm{n}}$ and $\phi \in \mathcal{C}$, then

$$
a K^{*} \widetilde{+}_{\phi} b L^{*}=\left(a K+_{\phi} b L\right)^{*}
$$

Proof. By the definition of Orlicz combination and $h\left(K^{*}, \cdot\right)=\frac{1}{\rho(K, \cdot)}$, we have, for every $u \in S^{n-1}$, let 
$\mathrm{K}_{\phi}=\mathrm{aK}+_{\phi} \mathrm{bL}$,

$$
\begin{aligned}
1 & =a \phi\left(\frac{h(\mathrm{~K}, \mathrm{u})}{\mathrm{h}\left(\mathrm{K}_{\phi}, \mathrm{u}\right)}\right)+b \phi\left(\frac{\mathrm{h}(\mathrm{L}, \mathrm{u})}{\mathrm{h}\left(\mathrm{K}_{\phi}, \mathrm{u}\right)}\right) \\
& =a \phi\left(\frac{\rho\left(\mathrm{K}_{\phi}^{*}, \mathrm{u}\right)}{\rho\left(\mathrm{K}^{*}, \mathrm{u}\right)}\right)+b \phi\left(\frac{\rho\left(\mathrm{K}_{\phi}^{*}, \mathrm{u}\right)}{\rho\left(\mathrm{L}^{*}, \mathrm{u}\right)}\right) .
\end{aligned}
$$

On the other hand, by the definition of Orlicz radial addition,

$$
1=a \phi\left(\frac{\rho\left(a K^{*} \widetilde{+}_{\phi} b L^{*}, u\right)}{\rho\left(K^{*}, u\right)}\right)+b \phi\left(\frac{\rho\left(a K^{*} \widetilde{+}_{\phi} b L^{*}, u\right)}{\rho\left(L^{*}, u\right)}\right) .
$$

Thus, from uniqueness of solution to the equation

$$
a \phi\left(\frac{f}{\rho\left(K^{*}, u\right)}\right)+b \phi\left(\frac{f}{\rho\left(L^{*}, u\right)}\right), f \in C\left(S^{n-1}\right),
$$

we conclude that $a K^{*} \widetilde{+}_{\phi} b L^{*}=\left(a K+_{\phi} b L\right)^{*}$.

When $\phi(t)=t^{p}, p \geqslant 1$, then $[a K+p b L]^{*}=a K^{*}+-p a L^{*}, a, b>0$.

Theorem 3.2. Let $\mathrm{K}, \mathrm{L} \in \mathcal{K}_{0}^{n}, \lambda \in(0,1)$ and $\phi \in \mathcal{C}$. Then

$$
1 \geqslant \lambda \phi\left(\frac{\left|\left[\lambda \mathrm{K}+_{\phi}(1-\lambda) \mathrm{L}\right]^{*}\right|^{\frac{1}{n}}}{\left|\mathrm{~K}^{*}\right|^{\frac{1}{n}}}\right)+(1-\lambda) \phi\left(\frac{\left|\left[\lambda \mathrm{K}+_{\phi}(1-\lambda) \mathrm{L}\right]^{*}\right|^{\frac{1}{n}}}{\left|\mathrm{~L}^{*}\right|^{\frac{1}{n}}}\right) .
$$

If $\phi$ is strictly convex, then equality holds if and only if $\mathrm{K}$ and $\mathrm{L}$ are dilates of each other.

Proof. Equation (1.2) implies that

$$
1 \geqslant a \phi\left(\left(\frac{\left|a K^{*} \widetilde{+}_{\phi} b L^{*}\right|}{\left|K^{*}\right|}\right)^{\frac{1}{n}}\right)+b \phi\left(\left(\frac{\left|a K^{*} \widetilde{+}_{\phi} b L^{*}\right|}{\left|L^{*}\right|}\right)^{\frac{1}{n}}\right) .
$$

Combination with Lemma 3.1, this gives the desired inequality. By the equality holds of (1.2), we know that, if $\phi$ is strictly convex, then equality holds if and only if $K$ and $L$ are dilates of each other.

Proof of Theorem 1.3. By Theorem 3.2, it follows that

$$
\begin{aligned}
1 & \geqslant \lambda \phi\left(\frac{\left|\left[\lambda \mathrm{K}+_{\phi}(1-\lambda) \mathrm{L}\right]^{*}\right|^{\frac{1}{n}}}{\left|\mathrm{~K}^{*}\right|^{\frac{1}{n}}}\right)+(1-\lambda) \phi\left(\frac{\left.\left|\left[\lambda \mathrm{K}+_{\phi}(1-\lambda) \mathrm{L}\right]^{*}\right|\right)^{\frac{1}{n}}}{\left|\mathrm{~L}^{*}\right|^{\frac{1}{n}}}\right) \\
& \geqslant \phi\left(\lambda \frac{\left|\left[\lambda \mathrm{K}+_{\phi}(1-\lambda) \mathrm{L}\right]^{*}\right|^{\frac{1}{n}}}{\left|\mathrm{~K}^{*}\right|^{\frac{1}{n}}}+(1-\lambda) \frac{\left|\left[\lambda \mathrm{K}+_{\phi}(1-\lambda) \mathrm{L}\right]^{*}\right|^{\frac{1}{n}}}{\left.\mid \mathrm{L}^{*}\right)^{\frac{1}{n}}} \mid,\right.
\end{aligned}
$$

which gives

$$
\phi^{-1}(1) \geqslant \lambda \frac{\left|\left[\lambda \mathrm{K}+_{\phi}(1-\lambda) \mathrm{L}\right]^{*}\right|^{\frac{1}{n}}}{\left|\mathrm{~K}^{*}\right|^{\frac{1}{n}}}+(1-\lambda) \frac{\left|\left[\lambda \mathrm{K}+_{\phi}(1-\lambda) \mathrm{L}\right]^{*}\right|^{\frac{1}{n}}}{\left|\mathrm{~L}^{*}\right|^{\frac{1}{n}}}
$$

thus

$$
\left|\left[\lambda \mathrm{K}+_{\phi}(1-\lambda) \mathrm{L}\right]^{*}\right|^{-\frac{1}{n}} \geqslant \lambda\left|\phi^{-1}(1) \mathrm{K}^{*}\right|^{-\frac{1}{n}}+(1-\lambda)\left|\phi^{-1}(1) \mathrm{L}^{*}\right|^{-\frac{1}{n}} .
$$

We now consider the function $f(x)=1 / x, x>0$, obviously, $f(x)$ is convex function, it follows that

which yields

$$
\begin{aligned}
\left|\left(\lambda K+_{\phi}(1-\lambda) L\right)^{*}\right|^{\frac{1}{n}} & \leqslant \frac{1}{\lambda\left|\phi^{-1}(1) K^{*}\right|^{-\frac{1}{n}}+(1-\lambda)\left|\phi^{-1}(1) L^{*}\right|^{-\frac{1}{n}}} \\
& \leqslant \frac{1}{\left[\left|\phi^{-1}(1) K^{*}\right|^{-\frac{1}{n}}\right]^{\lambda}\left[\left|\phi^{-1}(1) L^{*}\right|^{-\frac{1}{n}}\right]^{1-\lambda}},
\end{aligned}
$$

$$
\left|\left(\lambda \mathrm{K}+_{\phi}(1-\lambda) \mathrm{L}\right)^{*}\right| \leqslant\left|\phi^{-1}(1) \mathrm{K}^{*}\right|^{\lambda}\left|\phi^{-1}(1) \mathrm{L}^{*}\right|^{1-\lambda} .
$$

This completes the proof. 


\section{Acknowledgment}

We would like to thank the referees for valuable suggestions and comments that lead to improvement of the original manuscript.

\section{References}

[1] A. D. Aleksandrov, On the theory of mixed volumes.I. Extension of certain concepts in the theory of convex bodies, Mat. Sb., 2 (1937), 947-972. 1.1

[2] K. J. Böröczky, E. Lutwak, D. Yang, G. Zhang, The log-Brunn-Minkowski inequality, Adv. Math., 231 (2012), 19741997. 1.1

[3] S. Campi, P. Gronchi, The L ${ }^{p}$ Busemann-Petty centroid inequality, Adv. Math., 167 (2002), 128-141.

[4] S. Campi, P. Gronchi, On volume product inequalities for convex sets, Proc. Amer. Math. Soc., 134 (2006), $2393-2402$.

[5] W. J. Firey, Mean cross-section measures of harmonic means of convex bodies, Pacific J. Math., 11 (1961), 1263-1266. 1.1, 1.1

[6] R. J. Gardner, D. Hug, W. Weil, The Orlicz-Brunn-Minkowski theory: A general framework, additions, and inequalities, J. Differential Geom., 97 (2014), 427-476. 1.2

[7] C. Haberl, E. Lutwak, D. Yang, G. Zhang, The even Orlicz-Minkowski problem, Adv. Math., 224 (2010), $2485-2510$. $1.1,2$

[8] M. A. Hernández Cifre, J. Yepes Nicolás, On Brunn-Minkowski type Inequalities for polar bodies, J. Geometric Anal., 26 (2016), 143-155. 1.1, 1.1

[9] A.-J. Li, G. Leng, A new proof of the Orlicz Busemann-Petty centroid inequality, Proc. Amer. Math. Soc., 139 (2011), 1473-1481.

[10] E. Lutwak, Centroid Bodies and Dual Mixed Volumes, Proc. London Math. Soc., 60 (1990), 365-391. 1.1, 1.2

[11] E. Lutwak, The Brunn-Minkowski-Firey theory. I. Mixed volumes and the Minkowski problem, J. Differential Geom., 38 (1993), 131-150. 1.1, 1.2

[12] E. Lutwak, The Brunn-Minkowski-Firey Theory II: affine and geominimal surface area, Adv. Math., 118 (1996), $244-294$.

[13] E. Lutwak, V. Oliker, On the regularity of solutions to a generalization of the Minkowski problem, J. Differential Geom., 41 (1995), 227-246.

[14] E. Lutwak, D. Yang, G. Zhang, $\mathrm{L}_{\mathrm{p}}$-affine isoperimetric inequalities, J. Differential Geom., 56 (2000), 111-132.

[15] E. Lutwak, D. Yang, G. Zhang, Orlicz centroid bodies, J. Differential Geom., 84 (2010), 365-387. 1.1

[16] E. Lutwak, D. Yang, G. Zhang, Orlicz projection bodies, Adv. Math., 223 (2010), 220-242. 1.1

[17] E. Lutwak, G. Zhang, Blaschke Santaló inequalities, J. Differential Geom., 47 (1997), 1-16. 1.1

[18] R. Schneider, Convex Bodies: The Brunn-Minkowski Theory, Second expanded edition, Cambridge University Press, Cambridge, (2014). 1.1, 2

[19] D. Xi, H. Jin, G. Leng, The Orlicz Brunn-Minkowski inequality, Adv. Math., 260 (2014), 350-374. 1.2

[20] G. Y. Zhang, Centered Bodies and Dual Mixed Volumes, Tran. Amer. Math. Soc., 345 (1994), 777-801. 1.1

[21] B. Zhu, J. Zhou, W. Xu, Dual Orlicz-Brunn-Minkowski theory, Adv. Math., 264 (2014), 700-725. 1.2 